\title{
Stability analysis of COVID-19 model with fractional-order derivative and a delay in implementing the quarantine strategy
}

\author{
M. M. Hikal ${ }^{1,2} \cdot$ M. M. A. Elsheikh³ $\cdot$ W. K. Zahra ${ }^{1,4}$
}

Received: 14 January 2021 / Revised: 7 February 2021 / Accepted: 9 February 2021 /

Published online: 22 March 2021

(c) Korean Society for Informatics and Computational Applied Mathematics 2021

\begin{abstract}
This study presents methods of hygiene and the use of masks to control the disease. The zero basic reproduction number can be achieved by taking the necessary precautionary measures that prevent the transmission of infection, especially from uninfected virus carriers. The existence of time delay in implementing the quarantine strategy and the threshold values of the time delay that keeping the stability of the system are established. Also, it is found that keeping the infected people quarantined immediately is very important in combating and controlling the spread of the disease. Also, for special cases of the system parameters, the time delay can not affect the asymptotic behavior of the disease. Finally, numerical simulations have been illustrated to validate the theoretical analysis of the proposed model.
\end{abstract}

Keywords Stability analysis $\cdot$ SEIRUS covid-19 epidemic model $\cdot$ Caputo fractional derivative

W. K. Zahra

waheed.zahra@ejust.edu.eg

M. M. Hikal

manalhikal@yahoo.com

M. M. A. Elsheikh

mshikh_1999@yahoo.com

1 Departement of Engineering Physics and Mathematics, Faculty of Engineering, Tanta University, Tanta, Egypt

2 Department of Basic Sciences, Higher Institute of Engineering and Technology Kafrelsheikh, Kafr El Sheikh, Egypt

3 Departement of Mathematics, Faculty of Science, Menofia University, Shebin El-Koom, Egypt

4 Department of Mathematics, Institute of Basic and Applied Sciences, Egypt-Japan University of Science and Technology (E-JUST), New Borg El-Arab City 21934, Alexandria, Egypt 


\section{Introduction}

The COVID-19 pandemic is a hard test of the world system where it is a global health-economic shock that forced all the contries to face frightening risks. The coronavirus disease (COVID-19) is respiratory disease, caused by a novel coronavirus (SARS-CoV-2), with a similar genome to bat coronavirus [1]. The existence of COVID-19 has been noted firstly in China then widely spread in all the world countries. By the last of January 2020, the World Health Organization (WHO) considered the COVID-19 epidemic as a serious challenge to global health. The confirmed cases at March 2020 was 87,137, most of them in China with 2977 deaths [2]. However, on September 29, 2020, there are over 34 million infected people and 1.01 million dead cases in the world (3\%) [3]. On 14 December 2020 , the number of confirmed cases was 70,461,926 with 1,599,704 deaths, as declared by WHO [4].

Researchers have identified that COVID-19 is transimeted through the respiratory tract. And the main method in spreading the disease is human-to-human through droplets from infected individual during he cough, sneeze, or breathe. These droplets fall to the ground or surfaces and make them polluted. Also infection by the virouse can occure through breathing if one is very close to an infected person with Covid-19 disease. The clinical symptoms of COVID-19 patients are Fever, Cough, Dyspnea, Headache, Sore throat, Rhinorrhea and fatigue. The elderly and the persons having chronic diseases or immunodeficiency diseases are very weak in resisting COVID-19 disease.

Censolo and Morelli [5] monitored the impact of COVID-19 as one of the dangerous epidemics on social stability. They indicated that as long as the epidemic continues, the previous turmoil will compete with social grievances resulting from the spread of the epidemic to worsen the situation, and they considered that unifying governments is necessary to confront social instability resulting from the epidemic. Dias and Rathnayaka [6] presented studies on the disease transmission and recognition of its genome, analyzed and compared with other coronaviruses. Also, they identified the disease symptoms and developed methods to identify infected patients. But they stated that it will take a long time to develop a suitable vaccine for this disease. Renyi Zhang et al. [7] showed that the inadequate knowledge of virus transmission led to lose the ability in controlling the spread of the COVID-19 disease. And they concluded that transmission through air is highly ocure. Also, Harapan et al. [8], concluded that there are rapid advances in the information of the nature of COVID-19, so all countries should do their best to face the disease by saving mony and effort to improve the national laboratory system. The main methods to controlle the spread of COVID-19 are the social distancing by keeping at least 6 feets of distance between individuals, useing face masks, covering coughs and sneezes, washing hands, avoiding contact with infected people, and in case of symptoms, applying the advisiable medical protocole [9]. Tahir et al. [10] was concerned with the spread of MERS-CoV among the human by an agent known as the camel. They got the basic reproduction number $R_{0}$ of the model, and they gave the conditions for global stability of 
the system using the Lyapunov function. Then they considered a control strategy by using a mask to cover full body parts and medication to reduce the number of infected persons. Mohsen et al. [11] represented a COVID-19 model considering media coverage effects. The model has two equilibrium points; the COVID-19 free point which is locally asymptotically stable when $R_{0}<1$ and the COVID-19 equilibrium point that is asymptotically stable for $R_{0}>1$ by verifying the Liapunov function. Also, Victor Alexander Okhuese [12] considered a SEIRUS model describing the COVID-19 system. He evaluated the basic reproduction number and found that it is always less than unity, so the decline of secondary infections can be occured when all the countries obey the precautionary measures. Thus he advised that more effort should be done by the governments and health officials to decrease the spread of the disease especially in the case of the absence of a vaccine. The WHO has stated an incubation period for COVID-19 between 2 and 10 days and sometimes increases to be more than two weeks. So this long period may reflect double exposure [13]. Therefore, we build our system according to the fact that the infection by the disease can exist through the infected individuals and the exposed individuals who carry the novel coronavirus SARS-CoV-2 but are not infected. Also because the fractional calculus represents a generalization of differentiation and integration to non-integer orders, it is an excellent tool in describing physical and biological phenomena that depend on the previous history of the variables. Saeedian et al. [14], used the fractional calculus tool to observe the influence of memory effects on the dynamics of epidemiological models. Through Caputo's approach, they convert the differential equations of the standard SIR model to the fractional derivatives, in order to consider memory effects on the epidemic thresholds. For more details, we may recommend the reader to see [14].

Hence we present our model in fractional-order differential equations. And considering the following relations between the system's variables declared by the diagram in Fig. 1 that helps in describing our system. So the mathematical model is estimated by the following system of fractional order differential equations:

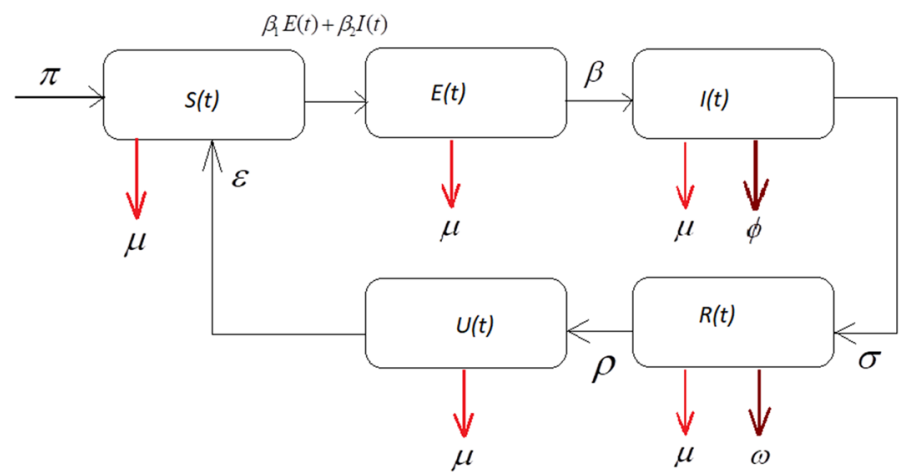

Fig. 1 Flow diagram of the presented novel model of COVID-19 


$$
\begin{aligned}
& D^{\alpha} S(t)=\pi-\mu S(t)+\varepsilon U(t)-S(t)\left(\beta_{1} E(t)+\beta_{2} I(t)\right), \\
& D^{\alpha} E(t)=S(t)\left(\beta_{1} E(t)+\beta_{2} I(t)\right)-\mu E(t)-\beta(t) E(t), \\
& D^{\alpha} I(t)=\beta(t) E(t)-(\sigma+\varphi+\mu) I(t), \\
& D^{\alpha} R(t)=\sigma I(t)-(\rho+\omega+\mu) R(t), \\
& D^{\alpha} U(t)=\rho R(t)-(\varepsilon+\mu) U(t),
\end{aligned}
$$

where $\quad \alpha \in(0,1], \beta(t)=\frac{\beta^{*} I(t)+\sigma R(t)+\rho U(t)}{N}, N=S+E+I+R+U$,

and $S(0)=S_{0}, E(0)=E_{0}, I(0)=I_{0}, R(0) \stackrel{N}{=} R_{0}, U(0)=U_{0}$.

The population is divided into five classes, the susceptible individuals class $S(t)$, exposed but not yet infectious class $E(t)$, infected class $I(t)$, infected class population quarantined $R(t)$ and recovered individuals $U(t)$. The system parameters all are positive. Let $\pi$ represents the new birth rate in a susceptible population, $\mu$ is the natural death rate of the population. Assuming $\varepsilon$ is the proportion of the recovered population being moved to susceptible class. The parameters $\beta_{1}$ and $\beta_{2}$ represent the rate of transmission of susceptibility to be exposed to individuals due to the infection from the transmission of the virus from the exposed and infected classes. In the absence of health awareness and educating individuals on precautionary measures towards illness, especially wearing the mask the values of $\beta_{1}$ and $\beta_{2}$ will increase. $\beta E$ is the portion of the exposed individuals to be infected. Let us consider that $\sigma I$ is the portion of the infected population to be infected population quarantined, $\rho R$ is the portion of the recovered individuals appropriate treatment and still being observed consequently belong to the recovered class. Assuming that $\varphi$ and $\omega$ are disease-induced death rates of the infected population not quarantined and infected receiving quarantined respectively.

Now we recall some basic definitions considering the fractional order of derivatives. Consider the system

$$
D^{\alpha} x(t)=f(x), \alpha \in(0,1], x \in R^{n},
$$

where $D^{\alpha}$ is the Caputo fractional derivative which is given in the following definition.

Definition 1.1 [15, 16] The Caputo fractional derivative is defined as

$$
D_{a}^{\alpha} f(x)=\frac{1}{\Gamma(n-\alpha)} \int_{a}^{x}(x-t)^{n-\alpha-1} f^{(n)}(t) d t, \quad n-1<\alpha \leq n .
$$

The properties of the solutions of the system (1.2) can be described by considering the stability behavior of its constant solutions. These constant solutions are determined by solving the system of algebraic equations $f\left(x^{*}\right)=0$.

Lemma 1.1 [17, 18] System (1.2) is locally asymptotically stable at the equilibrium point $x^{*}$ if each eigenvalue $\lambda$ of the Jacobian matrix of the system evaluated at $x^{*}$ lies in the stability region in the two-dimension plane which satisfies that. 


$$
|\arg \lambda|>\frac{\alpha \pi}{2}
$$

The main problem now is to locate the eigenvalues, so we need the following

Definition 1.2 [19] The discriminate $D(f)$ of $\underset{n}{\text { a }}$ polynomial $f(x)=x^{n}+a_{1} x^{n-1}+a_{2} x^{n-2}+\cdots+a_{n}$, is given by $D_{n}(f)=(-1)^{\frac{n(n-1)}{2}} R\left(f, f^{\prime}\right)$ where $f^{\prime}$ is the derivative of $f(x)$.

Let $g(x)=x^{l}+b_{1} x^{l-1}+b_{2} x^{l-2}+\ldots+b_{n}$, hence $R(f, g)$ is an $(n+l) \otimes(n+l)$ determinant.

The discriminate of a polynomial plays an important role to define the nature of the roots of $f(x)=0$. Let

$$
P(\lambda)=\lambda^{3}+a_{1} \lambda^{2}+a_{2} \lambda+a_{3}
$$

then

$$
D_{3}(P)=a_{1}^{2} a_{2}^{2}+18 a_{1} a_{2} a_{3}-4 a_{3} a_{1}^{3}-4 a_{2}^{3}-27 a_{3}^{2} .
$$

Theorem 1.1 [18] If the system (1.2) has a characteristic polynomial at $x^{*}$ defined by (1.5), then according to the sign of the discriminate $D_{3}(P)$ given by (1.6), condition (1.4) is satisfied in the following cases.

1. If $D_{3}(P)>0, a_{i}>0, i=1: 3, a_{1} a_{2}-a_{3}>0$ and $\alpha \in(0,1]$.

2. If $D_{3}(P)<0, a_{i} \geq 0, i=1: 3$ and $\alpha \in\left(\frac{2}{3}, 1\right]$.

3. If $D_{3}(P)<0, a_{i} \geq 0, i=1,2, a_{1} a_{2}=a_{3}$ and $\alpha \in(0,1]$.

$$
\begin{aligned}
& \text { If } P(\lambda)=\lambda^{4}+a_{1} \lambda^{3}+a_{2} \lambda^{2}+a_{3} \lambda+a_{4}, \text { then its discriminate is } \\
& \qquad \begin{aligned}
D_{4}(P)= & 18 a_{1}^{3} a_{2} a_{3} a_{4}-27 a_{1}^{4} a_{4}^{2}-4 a_{1}^{3} a_{3}^{3}-4 a_{1}^{2} a_{2}^{3} a_{4}+a_{1}^{2} a_{2}^{3} a_{3}^{2}+144 a_{1}^{2} a_{2} a_{3}^{2}-6 a_{1}^{2} a_{3}^{2} a_{4} \\
& -80 a_{1} a_{2}^{2} a_{3} a_{4}+18 a_{1} a_{2} a_{3}^{3}-192 a_{1} a_{2} a_{4}^{2}+16 a_{2}^{4} a_{4} \\
& -4 a_{2}^{3} a_{3}^{2}-128 a_{2}^{2} a_{4}^{2}+144 a_{1} a_{2}^{2} a_{4}-27 a_{3}^{4}+256 a_{4}^{3} .
\end{aligned}
\end{aligned}
$$

Let the characteristic equation has the form

$$
\lambda^{4}+a_{1} \lambda^{3}+a_{2} \lambda^{2}+a_{3} \lambda+a_{4}=0 .
$$

The following theorem gives important results in determining the stability properties of the system (1.2) that have the characteristic equation at the equilibrium point $x^{*}$ defined by Eq. (1.8).

Theorem 1.2 [20] Consider the determinants $\Delta_{i}, i=1: 3$ defined by. 


$$
\Delta_{1}=a_{2}, \Delta_{2}=\left|\begin{array}{cc}
a_{1} & 1 \\
a_{3} & a_{2}
\end{array}\right|, \Delta_{3}=\left|\begin{array}{ccc}
a_{1} & 1 & 0 \\
a_{3} & a_{2} & a_{1} \\
0 & a_{4} & a_{3}
\end{array}\right|,
$$

1. The equilibrium point $x^{*}$ is locally asymptotically stable for $\alpha=1$ if and only if $a_{4}>0$ and $\Delta_{i}>0, i=1: 3$.

2. If $D_{4}(P)>0, a_{1}>0$ and $a_{2}<0$, then the equilibrium point $x^{*}$ is unstable for $\alpha>\frac{2}{3}$.

3. If $D_{4}(P)\left\langle 0\right.$ and $\left.a_{i}\right\rangle 0, i=1: 4$, then the equilibrium point $x^{*}$ is locally asymptotically stable for $\alpha<\frac{1}{3}$. However if $D_{4}(P)<0, a_{1}\left\langle 0, a_{3}\left\langle 0\right.\right.$ and $\left.\left.a_{2}\right\rangle 0, a_{4}\right\rangle 0$ then the equilibrium point $x^{*}$ is unstable.

4. If $D_{4}(P)\left\langle 0, a_{i}\right\rangle 0, i=1: 4$ and $a_{2}=\frac{a_{1} a_{4}}{a_{3}}+\frac{a_{3}}{a_{1}}$, then the equilibrium point $x^{*}$ is locally asymptotically stable for all $\alpha \in(0,1)$.

5. The equilibrium point $x^{*}$ is locally asymptotically stable only if $a_{4}>0$.

This work is organized as follows: in Sect. 2, we evaluate the equilibrium points and the basic reproduction number of the system (1.1). Further, we discuss the boundedness of solutions of the system. In Sect. 3, we give sufficient conditions that guarantee the stability of the equilibrium points of the system. Moreover, in Sect. 4 , we discuss the effect of any delay in the treatment of the infectious individual on the stability of the system. In Sect. 5, we give some numerical examples to declare the obtained results using the non-standard finite difference method through Matlab programs. Finally, the conclusion is stated in Sect. 6.

\section{Equilibrium point of the model}

It is known that the equilibrium state of the endemic SEIRUS model is obtained by setting the left-hand sides of equations in (1.1) to zero. The constant solutions that will be considered are belong to $\subset\left(R^{+}\right)^{5}$ and evaluated by solving the system of algebraic equations:

$$
D^{\alpha} S(t)=D^{\alpha} E(t)=D^{\alpha} I(t)=D^{\alpha} R(t)=D^{\alpha} U(t)=0 .
$$

The equilibrium points are the free-disease equilibrium points (FDEP), $P_{0}$ and $P_{0}^{*}$

$$
\begin{aligned}
P_{0} \equiv\left(S_{0}, E_{0}, I_{0}, R_{0}, U_{0}\right) & =\left(\frac{\pi}{\mu}, 0,0,0,0\right), P_{0}^{*} \equiv\left(S_{0}^{*}, E_{0}^{*}, I_{0}^{*}, R_{0}^{*}, U_{0}^{*}\right) \\
& =\left(\frac{\mu}{\beta_{1}}, \frac{\pi \beta_{1}-\mu^{2}}{\mu \beta_{1}}, 0,0,0\right),
\end{aligned}
$$

and the infected equilibrium point $P_{1} \equiv\left(S_{1}, E_{1}, I_{1}, R_{1}, U_{1}\right)$ where: 


$$
\begin{aligned}
& E_{1}=\frac{N(\sigma+\varphi+\mu)}{\beta^{*}+\frac{\sigma^{2}}{\rho+\omega+\mu}+\frac{\sigma \rho^{2}}{(\varepsilon+\mu)(\rho+\omega+\mu)}}, S_{1}=\frac{\pi+\frac{I_{1} \varepsilon \rho \sigma}{(\varepsilon+\mu)(\rho+\omega+\mu)}}{\mu+\beta_{1} E_{1}+\beta_{2} I_{1}}, \\
& R_{1}=\frac{\sigma}{\rho+\omega+\mu} I_{1}, U_{1}=\frac{\sigma \rho}{(\varepsilon+\mu)(\rho+\omega+\mu)} I_{1} .
\end{aligned}
$$

where $I_{1}$ is the positive root of the quadratic equation $c_{1} I_{1}^{2}+c_{2} I_{1}+c_{3}=0$ if exist,

$$
\begin{aligned}
& c_{1}=\beta_{2}(\sigma+\varphi+\mu)\left[1-\frac{\varepsilon \sigma \rho}{(\varepsilon+\mu)(\rho+\omega+\mu)(\sigma+\varphi+\mu)}\right], \\
& c_{2}=\beta_{1} E_{1}(\sigma+\varphi+\mu)\left[1-\frac{\varepsilon \sigma \rho}{(\varepsilon+\mu)(\rho+\omega+\mu)(\sigma+\varphi+\mu)}\right]+\mu\left(\beta_{2} E_{1} \sigma+\varphi+\mu\right)-\pi \beta_{2}, \\
& c_{3}=\mu^{2} E_{1}\left(1-R_{0}+\frac{\beta_{1} E_{1}}{\mu}\right),
\end{aligned}
$$

There are two considerable especial cases, the first when neglecting the transmission of the disease from the exposed individuals, i.e. considering $\beta_{1}=0$. Hence the equilibrium points of the system are only two points, the free disease point $P_{0}=\left(\frac{\pi}{\mu}, 0,0,0,0\right)$ and the infected equilibrium point $P_{1}^{*} \equiv\left(S_{1}^{*}, E_{1}^{*}, I_{1}^{*}, R_{1}^{*}, U_{1}^{*}\right)$ where:

$$
\begin{gathered}
E_{1}^{*}=\frac{I_{1}^{*}}{\mu}\left[\frac{\beta_{2}}{\mu+\beta_{2} I_{1}^{*}}\left(\pi+\frac{\varepsilon \sigma \rho I_{1}^{*}}{(\varepsilon+\mu)(\rho+\omega+\mu)}\right)-(\sigma+\varphi+\mu)\right], \\
S_{1}^{*}=\frac{1}{\mu+\beta_{2} I_{1}^{*}}\left(\pi+\frac{\varepsilon \sigma \rho I_{1}^{*}}{(\varepsilon+\mu)(\rho+\omega+\mu)}\right), \\
R_{1}^{*}=\frac{\sigma I_{1}^{*}}{(\rho+\omega+\mu)}, U_{1}^{*}=\frac{\sigma \rho I_{1}^{*}}{(\varepsilon+\mu)(\rho+\omega+\mu)}
\end{gathered}
$$

where $I_{1}^{*}$ is the root of the quadratic equation $d_{1} I_{1}^{* 2}+d_{2} I_{1}^{*}+d_{3}=0$ when

$$
\begin{aligned}
& d_{2}<-2 \mu(\sigma+\varphi+\mu) \sqrt{\beta_{2} l\left(1-\frac{\varepsilon \sigma \rho}{(\varepsilon+\mu)(\rho+\omega+\mu)(\sigma+\varphi+\mu)}\right)}, \text { where } \\
& d_{1}=\beta_{2} l\left[(\sigma+\varphi+\mu)-\frac{\varepsilon \sigma \rho}{(\varepsilon+\mu)(\rho+\omega+\mu)}\right], \quad d_{2}=\mu(\sigma+\varphi+\mu)\left(l+\beta_{2}\right)-l \pi \beta_{2}, \\
& d_{3}=\mu^{2}(\sigma+\varphi+\mu), \quad l=\beta^{*}+\frac{\sigma^{2}}{(\rho+\omega+\mu)}+\frac{\sigma \rho^{2}}{(\varepsilon+\mu)(\rho+\omega+\mu)} .
\end{aligned}
$$

The second special case is when the function $\beta(t)$ be a constant value $\beta$, then the equilibrium points of the system are the free disease point $P_{0}=\left(\frac{\pi}{\mu}, 0,0,0,0\right)$ and the infected equilibrium point $\overline{P_{1}} \equiv\left(\overline{S_{1}}, \overline{E_{1}}, \overline{I_{1}}, \overline{R_{1}}, \overline{U_{1}}\right)$, where: 


$$
\begin{aligned}
& \overline{I_{1}}=\frac{\overline{R_{0}}-1}{\overline{R_{0}}} \pi \beta \frac{(\varepsilon+\mu)(\rho+\omega+\mu)}{(\beta+\mu)(\varepsilon+\mu)(\rho+\omega+\mu)(\sigma+\varphi+\mu)-\beta \varepsilon \sigma \rho}, \overline{E_{1}}=\frac{(\sigma+\varphi+\mu)}{\beta} \overline{I_{1}}, \\
& \overline{S_{1}}=\frac{1}{\mu}\left[\pi+\overline{I_{1}}\left(\frac{\varepsilon \sigma \rho}{(\varepsilon+\mu)(\rho+\omega+\mu)}-\frac{(\beta+\mu)(\sigma+\varphi+\mu)}{\beta}\right)\right], \quad \overline{R_{1}}=\frac{\sigma \overline{I_{1}}}{(\rho+\omega+\mu)}, \\
& \overline{U_{1}}\left(\frac{\sigma \rho \overline{I_{1}}}{(\varepsilon+\mu)(\rho+\omega+\mu)}\right)
\end{aligned}
$$

Now, we will study the Boundedness of system (1.1) by examining the total population that expressed by the summation $N(t)$, let $N(t)=S(t)+E(t)+I(t)+R(t)+U(t)$, the fractional-order derivative of the function $N(t)$ is defined by: $D^{\alpha} N(t)=D^{\alpha} S(t)+D^{\alpha} E(t)+D^{\alpha} I(t)+D^{\alpha} R(t)+D^{\alpha} U(t)$. Substituting from Eq. (1.1), we have:

$$
D^{\alpha} N(t)+\mu N(t) \leq \pi
$$

Applying Laplace transform for both sides of the inequality (2.8), we have:

$$
N(s)=\frac{\pi}{s} \frac{1}{s^{\alpha}+\mu}+\frac{N^{*}}{s^{\alpha}+\mu},
$$

where $N^{*}$ is the initial condition, and applying inverse Laplace transform, we get:

$$
N(t) \leq N^{*} t^{\alpha-1} E_{\alpha, \alpha}\left(-\mu t^{\alpha}\right)+\pi \int_{0}^{t}(t-\tau)^{\alpha-1} E_{\alpha, \alpha}\left(-\mu(t-\tau)^{\alpha}\right) d \tau .
$$

Using the definition and properties of Mittage Lefler function, we can write:

$$
N(t) \leq N^{*} t^{\alpha-1} E_{\alpha, \alpha}\left(-\mu t^{\alpha}\right)+\pi t^{\alpha} E_{\alpha, \alpha-1}\left(-\mu t^{\alpha}\right) .
$$

Hence, we note that $N(t)$ is a bounded function for any time $t \geq 0$. So from the above discussion, we can give the following theorem.

Theorem 2.1 Any solution of system (1.1) with bounded initial conditions is uniformly bounded.

It is very useful to determine the basic reproduction number that plays an important role in spreading the disease and studying the stability behavior of the epidemic model. To compute the basic reproductive number of the system (1.1), we follow [21-25] by using the next-generation method. Hence we can rearrange system (1.1) to have the form:

$$
\dot{\psi}(t)=\eta(\psi)-\xi(\psi), \text { where } \psi=(E, I, R, U, S)^{T} \text { and }
$$




$$
\eta=\left[\begin{array}{c}
\left(\beta_{1} E+\beta_{2} I\right) S \\
0 \\
0 \\
0 \\
0
\end{array}\right], \quad \xi=\left[\begin{array}{c}
(\mu+\beta) E \\
(\sigma+\varphi+\mu) I-\beta E \\
(\rho+\omega+\mu) R-\sigma I \\
(\varepsilon+\mu) U-\rho R \\
\left(\mu+\beta_{1} E+\beta_{2} I\right) S-\varepsilon U-\pi
\end{array}\right] .
$$

The Jacobian matrices of $\eta$ and $\xi$ at the free- disease equilibrium point (FDEP) are:

$$
J_{\eta}=\left[\begin{array}{ll}
F & 0 \\
0 & 0
\end{array}\right], \quad J_{\xi}=\left[\begin{array}{cc}
V & 0 \\
F_{1} & F_{2}
\end{array}\right]
$$

where

$$
F=\left[\begin{array}{cc}
\frac{\beta_{1} \pi}{\mu} & \frac{\beta_{2} \pi}{\mu} \\
0 & 0
\end{array}\right], V=\left[\begin{array}{cc}
\mu & 0 \\
0 & \sigma+\varphi+\mu
\end{array}\right], F_{1}=\left[\begin{array}{cc}
0 & -\sigma \\
0 & 0 \\
\frac{\beta_{1} \pi}{\mu} & \frac{\beta_{2} \pi}{\mu}
\end{array}\right], F_{2}=\left[\begin{array}{ccc}
\rho+\omega+\mu & 0 & 0 \\
-\rho & \varepsilon+\mu & 0 \\
0 & -\varepsilon & \mu
\end{array}\right] .
$$

The spectral radius of the matrix $\left(F V^{-1}\right)$ gives the values of the basic reproduction number $R_{0}$ in the form:

$$
R_{0}=\frac{\beta_{1} \pi}{\mu^{2}}
$$

In the case, when there is no virus transmission from the exposed individuals, $\beta_{1}=0$, the reproduction number will be zero, i.e.

$$
\check{R}_{0}=0 \text {. }
$$

In this case, where there is no incidence rate in the recovered population, the secondary infection by patients with COVID-19 who have been clinically declared negative and are free from the virus. So the virus is completely cleared from the system. This means that although there exists currently no clinical medicine for the cure of COVID-19, there is a high chance of zero cases of reinfection after clinical recovery from the virus. Also when $\beta(t) \equiv \beta$ is a constant value, the reproduction number has the form:

$$
\overline{R_{0}}=\frac{\pi}{\mu} \frac{\left[\beta_{1}(\sigma+\varphi+\mu)+\beta \beta_{1}\right]}{\mu+\beta(\sigma+\varphi+\mu)} .
$$

We can summarize the relation between the equilibrium points of the system (1.1) and the reproduction number in the following theorem.

Theorem 2.2 System (1.1) has the free-disease equilibrium point (FDEP) $P_{0}=\left(\frac{\pi}{\mu}, 0,0,0,0\right)$ when $R_{0} \leq 1$ and if $R_{0}>1$, then the equilibrium points are $P_{0}^{*}=\left(\frac{\mu}{\beta_{1}}, \frac{\pi \beta_{1}-\mu^{2}}{\mu \beta_{1}}, 0,0,0\right)$ in addition to $P_{1}\left(S_{1}, E_{1}, I_{1}, R_{1}, U_{1}\right)$ defined in (2.3), if the 
equation $c_{1} I_{1}^{2}+c_{2} I_{1}+c_{3}=0$ with coefficients $c_{i}, i=1: 3$ explained in (2.4), has a real positive root. If $\beta_{1}=0$, the equilibrium points are $P_{0}$ and $P_{1}^{*}$ defined by $(2.5$, 2.6) while if $\beta(t)=\beta$, the equilibrium points are $P_{0}$ and $\overline{P_{1}}$ defined by (2.7).

\section{Stability behavior of the equilibrium points}

The reduction system of (1.1) can be obtained by replacing the variable, $S(t)$ from the equation

$$
N(t)=S(t)+E(t)+I(t)+R(t)+U(t),
$$

then we have:

$$
\begin{aligned}
& D^{\alpha} E(t)=\left(N-(E(t)+I(t)+R(t)+U(t))\left(\beta_{1} E(t)+\beta_{2} I(t)\right)-\mu E(t)-\beta(t) E(t),\right. \\
& D^{\alpha} I(t)=\frac{E(t)}{N}\left(\beta^{*} I(t)+\sigma R(t)+\rho U(t)\right)-(\sigma+\varphi+\mu) I(t), \\
& D^{\alpha} R(t)=\sigma I(t)-(\sigma+\omega+\mu) R(t), \\
& D^{\alpha} U(t)=\sigma R(t)-(\varepsilon \mu) U(t) .
\end{aligned}
$$

And the equilibrium points of the system (3.2) are $P_{0}{ }^{\prime} \equiv\left(E_{0}, I_{0}, R_{0}, U_{0}\right)=(0,0,0,0)$, when $R_{0} \leq 1$ and $P_{0}^{* \prime}=\left(\frac{\pi \beta_{1}-\mu^{2}}{\mu \beta_{1}}, 0,0,0\right)$ and $P_{1}{ }^{\prime} \equiv\left(E_{1}, I_{1}, R_{1}, U_{1}\right)$ (if $P_{1}{ }^{\prime} \in \Gamma \subset R^{+4}$ ) when $R_{0}>1$. The stability behavior of the system (1.1) is determined by studying the stability of the system (3.2). The nature of its equilibrium points is displayed now. We begin with the (FDEP $P_{0}^{\prime}$ ), so the Jacobian matrix of system (3.2) at $P_{0}$ is:

$$
J\left(P_{0}^{\prime}\right)=\left[\begin{array}{llll}
\beta_{1} N-\mu & \beta_{2} N & 0 & 0 \\
0 & -(\sigma+\varphi+\mu) & 0 & 0 \\
0 & \sigma & (\rho+\omega+\mu) & 0 \\
0 & 0 & \rho & -(\varepsilon+\mu)
\end{array}\right]
$$

and the characteristic equation of $J\left(P_{0}^{\prime}\right)$ is:

$$
\left(\lambda-\frac{\pi \beta_{1}}{\mu}+\mu\right)(\lambda+\varepsilon+\mu)(\lambda+(\sigma+\varphi+\mu))(\lambda+(\rho+\omega+\mu))=0
$$

Theorem 3.1 The free disease equilibrium point $\stackrel{\prime}{P}_{0}$ of the system (3.2) is globally asymptotically stable if $R_{0}<1$ and unstable if $R_{0}>1$ for $\alpha \in(0,1]$.

Proof The characteristic Eq. (3.4) has the following eigenvalues: 


$$
\lambda_{1}=\frac{\pi \beta_{1}-\mu^{2}}{\mu}, \lambda_{2}=-(\varepsilon+\mu), \lambda_{3}=-(\sigma+\varphi+\mu), \lambda_{4}=-(\rho+\omega+\mu) .
$$

All the eigenvalues have negative real-valued when $R_{0}<1$ so $\stackrel{\prime}{P_{0}}$ (and als $\rho P_{0}$ ) is locally asymptotically stable, and also when $R_{0}<1$, the equilibrium point $P_{0}$ is the only critical point of the system. Then $P_{0}$ is globally asymptotically stable. But if $R_{0}>1$, we note that $\lambda_{1}$ has a positive real value and consequently $P_{0}$ is unstable.

\section{Remarks}

1. The above result shows that the basic reproduction number $R_{0}$ controls the stability properties of the FDEP $P_{0}$ of the system (1.1).

2. When $\beta(t)=\beta$ the FDEP $P_{0}$ of (1.1) is globally asymptotically stable when $R_{0} \leq 1$ and locally asymptotically stable for $\beta_{1}=0$.

When $R_{0}>1$, system (3.2) has two equilibrium points ${\stackrel{\prime}{P_{0}^{*}}}_{0}$ and $\stackrel{\prime}{P_{1}}$. We first study the stability analysis at $P_{0}^{* \prime}$. In this case, the Jacobian matrix is

$$
J\left(P_{0}^{* \prime}\right)=\left[\begin{array}{llll}
\beta_{1} S_{0}^{*}-\mu-\beta_{1} E_{0}^{*} & \beta_{2} S_{0}^{*}-\beta_{1} E_{0}^{*}-\beta^{*} \frac{E_{0}^{*}}{N} & \beta_{1} E_{0}^{*}-\sigma \frac{E_{0}^{*}}{N} & -\beta_{1} E_{0}^{*}-\rho \frac{E_{0}^{*}}{N} \\
0 & \beta^{*} \frac{E_{0}^{*}}{N}-(\sigma+\varphi+\mu) & \sigma \frac{E_{0}^{*}}{N} & \rho \frac{E_{0}^{*}}{N} \\
0 & \sigma & -(\rho+\omega+\mu) & 0 \\
0 & 0 & \rho & -(\varepsilon+\mu)
\end{array}\right],
$$

and so the characteristic equation of $J\left(\begin{array}{c}1 \\ P^{*} \\ 0\end{array}\right)$ is

$$
\left(\lambda+\mu+2 \beta_{1} E_{0}^{*}-\beta_{1} N\right)\left(\lambda^{3}+a_{1} \lambda^{2}+a_{2} \lambda+a_{3}\right)=0,
$$

where:

$$
\begin{aligned}
& a_{1}=\varepsilon+3 \mu+\rho+\omega+\sigma+\varphi-\beta^{*} \frac{E_{0}^{*}}{N} \\
& a_{2}=(\varepsilon+\mu)(\rho+\omega+\mu)+(\sigma+\varphi+\mu)(\rho+\omega+\varepsilon+2 \mu)-\frac{E_{0}^{*}}{N}\left(\sigma^{2}+\beta^{*}(\varepsilon+\rho+\omega+2 \mu)\right. \\
& a_{3}=(\varepsilon+\mu)(\rho+\omega+\mu)(\sigma+\varphi+\mu)-\frac{E_{0}^{*}}{N}\left(\rho \sigma^{2}+\sigma^{2}(\varepsilon+\mu)+\beta^{*}(\varepsilon+\mu)(\rho+\omega+\mu)\right)
\end{aligned}
$$

Theorem 3.2 The equilibrium point ${ }^{\prime} P_{0}^{*}$ of the system (3.2) is locally asymptotically stable in the following cases:

1. If $D_{3}(P)>0, \alpha \in(0,1]$ and $\frac{1}{R_{0}}>1-m, m=\min \left\{m_{1}, m_{3}, m_{4}\right\}$.

2. If $D_{3}(P)<0, \alpha \in\left(\frac{2}{3}, 1\right]$ and $\frac{1}{R_{0}}>1-m^{*}, m^{*}=\min \left\{m_{1}, m_{2}, m_{3}\right\}$. 
3. If $D_{3}(P)<0, \alpha \in(0,1]$ and $\frac{1}{R_{0}}>1-\stackrel{\prime}{m}, \stackrel{\prime}{m}=\min \left\{m_{1}, m_{2}\right\}$ and $\frac{1}{R_{0}}=1-m_{4}$.

Else $P_{0}^{*}$ is unstable. Where $D_{3}(P)$ is defined by (1.6),

$$
\begin{aligned}
& m_{1}=\frac{a+b+c}{\beta^{*}}, m_{2}=a b+\frac{c(a+b)}{\sigma^{2}+\beta^{*}(a+b)}, m_{3}=\frac{a b c}{\rho \sigma^{2}+b \sigma^{2}+a b \beta^{*}}, \\
& m_{4}=0.5\left(d-\sqrt{d^{2}-\frac{4(a+b)(c(a+b+c)+a b)}{\beta^{*}\left((a+b) \beta^{*}+\sigma^{2}\right)}}\right)
\end{aligned}
$$

and

$$
a=\rho+\omega+\mu, b=\varepsilon+\mu, c=\sigma+\varphi+\mu .
$$

Proof The eigenvalues of the characteristic Eq. (3.6) are.

$$
\lambda_{1}=-\left(\mu+2 \beta_{1} E_{0}^{*}-\beta_{1} N\right)=-\frac{\left(\beta_{1} \pi-\mu^{2}\right)}{\mu}<0,
$$

when $R_{0}>1$ and the roots of the equation are

$$
\lambda^{3}+a_{1} \lambda^{2}+a_{2} \lambda+a_{3}=0
$$

with the coefficients $a_{i}, i=1: 3$ defined by (3.7).

Considering the discriminant $D_{3}(P(\lambda)$ ) displayed in (1.6). For case (i) if $D_{3}(P)>0$ then the sufficient conditions for locally asymptotically stability of $P_{0}^{*}$ are.

$a_{1}>0, a_{3}>0$ and $a_{1} a_{2}-a_{3}>0$.

By substituting from (3.7) and simplifying the three inequalities using (3.11), the three inequalities take the forms: $\frac{1}{R} 0>1-m_{1}, \frac{1}{R} 0>1-m_{3}$ and $\frac{1}{R} 0>1-m_{4}$. Also, condition (3.8) guarantees these inequalities.

Similarly, we can apply Theorem 1.1 to prove the cases (ii) and (iii).

, Now we are interested in the properties of the infection equilibrium point $P_{1} \equiv\left(E_{1}, I_{1}, R_{1}, U_{1}\right)$ defined by (2.3) and (2.4), to estimate the future of the disease. Consider the characteristic polynomial of $J\left(\begin{array}{c}1 \\ P_{1}\end{array}\right)$ as

$$
P(\lambda)=\lambda^{4}+b_{1} \lambda^{3}+b_{2} \lambda^{2}+b_{3} \lambda+b_{4},
$$

where 


$$
\begin{aligned}
b_{1} & =D_{1}+D_{2}+D_{3}, \\
b_{2} & =D_{1}\left(D_{2}+D_{3}\right)+D_{2} D_{3}+(\varepsilon+\mu)(\rho+\omega+\mu)+\beta\left(\beta_{1} E_{1}+\beta_{2} I_{1}+\frac{\beta^{*} E_{1}}{N}-\beta_{2} S_{1}\right), \\
b_{3} & =D_{1}\left((\varepsilon+\mu)(\rho+\omega+\mu)+D_{2}(\sigma+\varphi+\mu)-\frac{E_{1}}{N}\left(\beta^{*} D_{2}+\sigma^{2}\right)\right) \\
& +\beta\left(\sigma\left(\beta_{1} E_{1}+\beta_{2} I_{1}+\frac{\sigma E_{1}}{N}\right)+D_{2}\left(\beta_{1} E_{1}+\beta_{2} I_{1}+\frac{\beta^{*} E_{1}}{N}-\beta_{2} S_{1}\right),\right. \\
b_{4} & =\left(\beta_{1} E_{1}+\beta_{2} I_{1}\right)((\varepsilon+\mu)(\sigma+\rho+\omega+\mu)+\sigma \rho) \\
& +\beta(\varepsilon+\mu)(\rho+\omega+\mu)\left(\mu+\sigma+\varphi-\beta_{2} S_{1}\right), \\
D_{1} & =\beta_{1} E_{1}+\beta_{2} I_{1}+\beta+\mu-\beta_{1} S_{1}, D_{2}=\varepsilon+2 \mu+\rho+\omega, D_{3}=\mu+\sigma+\varphi-\frac{\beta^{*} E_{1}}{N} .
\end{aligned}
$$

We summarize local stability $P_{1}^{\prime}$ in the following theorem.

Theorem 3.3 The epidemic point $\stackrel{\prime}{P}_{1}$ of the system (3.2) is asymptotically stable if the parameters satisfy the following conditions.

1. for $\alpha=1, \varphi_{1}<\beta \beta_{2} S_{1}<\varphi^{*}, \varphi^{*}=\min \left\{\varphi_{3}, \varphi_{4}\right\}$ and $b_{1} b_{2}-b_{3}>\frac{b_{1}^{2} b_{4}}{b_{3}}$,

2. for $\alpha<\frac{1}{3}, D_{4}(P)<0, \varphi_{1}<\beta \beta_{2} S_{1}<\varphi^{* *}, \varphi^{* *}=\min \left\{\varphi_{2}, \varphi^{*}\right\}$,

3. for $\alpha \in(0,1)$, when conditions (3.17) is satisfied and $b_{1}\left(b_{2} b_{3}-b_{1} b_{4}\right)-b_{3}^{2}=0$

Where $b_{i}, i=1: 4$ are defined in (3.15),

$$
\begin{aligned}
& D_{4}(P)= 18 b_{1}^{3} b_{2} b_{3} b_{4}-27 b_{1}^{4} b_{2}^{2}-4 b_{1}^{3} b_{3}^{3}-4 b_{1}^{2} b_{2}^{3} b_{4}+b_{1}^{2} b_{2}^{3} b_{3}+144 b_{1}^{2} b_{2} b_{3}^{2} \\
&-80 b_{1} b_{2}^{2} b_{3} b_{4}-6 b_{1}^{2} b_{3}^{2} b_{4}+18 b_{1} b_{2} b_{3}^{3}-192 b_{1} b_{2} b_{4}^{2}+16 b_{2}^{4} b_{4}-4 b_{2}^{3} b_{3}^{2} \\
&-128 b_{2}^{2} b_{4}^{2}+144 b_{1} b_{2}^{2} b_{4}-27 b_{3}^{4}+256 b_{4}^{3} \\
& \varphi_{1}=\frac{\beta E_{1}}{I_{1}}\left(\frac{\beta^{*} E_{1}}{N}-\left(D_{2}+\sigma+\varphi+\mu+\beta_{1} E_{1}+\beta_{2} I_{1}\right)\right), \\
& \varphi_{2}=D_{1}\left(D_{2}+D_{3}\right)+(\varepsilon+\mu)(\rho+\omega+\mu)+D_{2} D_{3}+\beta\left(\beta_{1} E_{1}+\beta_{2} I_{1}+\frac{\beta^{*} E_{1}}{N}\right), \\
& \varphi_{3}=\frac{D_{1}}{D_{2}}\left((\varepsilon+\mu)(\rho+\omega+\mu)+D_{2}(\sigma+\varphi+\mu)-\frac{E_{1}}{N} \beta^{*} D_{2}+\sigma^{2}\right), \\
& \varphi_{4}=\frac{\left(\beta_{1} E_{1}+\beta_{2} I_{1}\right)((\varepsilon+\mu)(\rho+\omega+\mu)+\sigma \rho)+\beta(\varepsilon+\mu)(\rho+\omega+\mu)(\sigma+\varphi+\mu)}{(\varepsilon+\mu)(\rho+\omega+\mu)} .
\end{aligned}
$$

Proof The proof is similar to the proof of theorem 3.2. 


\section{Effect of a delay in implementing the quarantine for Corona patients}

Now, we discuss the existence of a delay in implementing the quarantine for Corona patients, the analysis that studies the stability of the system. Since the system (3.2) with time delaying $\tau \geq 0$ has the forms,

$$
\begin{aligned}
& D^{\alpha} E(t)=\left(N-(E(t)+I(t)+R(t)+U(t))\left(\beta_{1} E(t)+\beta_{2} I(t)\right)-\mu E(t)\right. \\
& -\frac{E(t)}{N}\left(\beta^{*} I(t)+\sigma R(t)+\rho U(t)\right) \\
& D^{\alpha} I(t)=\frac{E(t)}{N}\left(\beta^{*} I(t)+\sigma R(t)+\rho U(t)\right)-(\varphi+\mu) I(t)-\sigma I(t-\tau), \\
& D^{\alpha} R(t)=\sigma I(t-\tau)-(\sigma+\omega+\mu) R(t), \\
& D^{\alpha} U(t)=\sigma R(t)-(\varepsilon+\mu) U(t),
\end{aligned}
$$

when $R_{0}<1$ and $\alpha \in(0,1]$, the free disease equilibrium ${ }^{\prime} P_{0}$ is globally asymptotically stable when $\tau=0$ and the question now for $\tau>0$, is $P_{0}$ still asymptotically stable or becomes unstable?

For answering, the eigenvalues of the system (4.1) at the point $\stackrel{\prime}{P}_{0}$ must be obtained for $\tau>0$ and decide if there lie in the stable region or not.

Theorem 4.1 The FDEP $\stackrel{\prime}{P_{0}}$ of the system (4.1) is unstable for $\tau \geq \tau_{1}$ when $R_{0}<1, \alpha \in(0,1]$, if.

$$
\varphi+\mu<\sigma
$$

where

$$
\tau_{1}=\left[\begin{array}{ll}
\left(\frac{\frac{\pi}{2}+\cos ^{-1} \frac{\varphi+\mu}{\sigma}}{\sqrt{\sigma^{2}-(\varphi+\mu)^{2}}}\right) & \text { for } \quad \alpha=1, \\
-\left(\frac{1}{2 r \cos \left(\frac{\alpha \pi}{2}\right)} \ln \frac{\left(r^{2}+2 r(\varphi+\mu) \cos \left(\frac{\alpha \pi}{2}\right)+(\varphi+\mu)^{2}\right)}{\sigma^{2}}\right) & \text { for } \quad \alpha \in(0,1) .
\end{array}\right]
$$

Proof The Jacobian matrix of system (4.1) at ${ }^{\prime} P_{0}$ is

$$
J\left(P_{0}^{\prime}\right)=\left[\begin{array}{llll}
\beta_{1} N-\mu & \beta_{2} N & 0 & 0 \\
0 & -(\varphi+\mu)-\sigma e^{-\lambda \tau} & 0 & 0 \\
0 & \sigma e^{-\lambda \tau} & -(\rho+\omega+\mu) & 0 \\
0 & 0 & \rho & -(\varepsilon+\mu)
\end{array}\right],
$$

and the eigenvalues of $J\left(P_{0}{ }^{\prime}\right)$ are $\lambda_{1}=\frac{\pi \beta_{1}-\mu^{2}}{\mu}, \lambda_{2}=-(\varepsilon+\mu), \lambda_{3}=-(\rho+\omega+\mu)$, which are real negative values and the fourth eigenvalue is the solution of the nonlinear equation

$$
\lambda+(\varphi+\mu)+\sigma e^{-\lambda \tau}=0 .
$$


If there is a positive value of the time delay $\tau_{1}$ that makes the eigenvalue of Eq. (4.5) does not satisfy condition (1.4), the system becomes unstable at $P_{0}$ for $\tau \geq \tau_{1}$. The critical value of the eigenvalue is $\lambda=r e^{i \frac{\alpha \pi}{2}}$.

Substituting in Eq. (4.5) and using condition (4.2), we find that $\tau_{1}$ exists only when $\sigma<\varphi+\mu$ and its value is defined by (4.3).

Considering the equilibrium points $P_{0}^{*}$ when $R_{0} \geqslant 1$, and where it is locally asymptotically stable for $\tau=0$, the Jacobian matrix at $P_{0}^{*}$ for $\tau>0$ is:

$$
J\left(\begin{array}{c}
\prime \\
P_{0}^{*}
\end{array}\right)=\left[\begin{array}{llll}
\beta_{1}\left(N-E_{0}^{*}\right)-\mu-\beta_{1} E_{0}^{*} & \beta_{2} S_{0}^{*}-\beta_{1} E_{0}^{*}-\beta^{*} \frac{E_{0}^{*}}{N} & \beta_{1} E_{0}^{*}-\sigma \frac{E_{0}^{*}}{N} & -\beta_{1} E_{0}^{*}-\rho \frac{E_{0}^{*}}{N} \\
0 & \beta^{*} \frac{E_{0}^{*}}{N}-\left(\sigma e^{-\lambda \tau}+\varphi+\mu\right) & \sigma \frac{E_{0}^{*}}{N} & \rho \frac{E_{0}^{*}}{N} \\
0 & \sigma e^{-\lambda \tau} & -(\rho+\omega+\mu) & 0 \\
0 & 0 & \rho & -(\varepsilon+\mu)
\end{array}\right],
$$

the eigenvalues of the Jacobian matrix (4.6) are $\lambda_{1}=-\frac{\pi \beta_{1}-\mu^{2}}{\mu}$ and the roots of the equation

$$
\begin{aligned}
& \lambda^{3}+\left(g_{1}+g_{2}\right) \lambda^{2}+\sigma \lambda^{2} e^{-\lambda \tau}+\left(g_{1} g_{2}+g_{3}\right) \lambda+\sigma\left(g_{1}-\sigma \frac{E_{0}^{*}}{N}\right) \lambda e^{-\lambda \tau} \\
& \quad+\sigma\left(g_{3}-\frac{E_{0}^{*}}{N}\left(\rho^{2}+\sigma(\varepsilon+\mu)\right) e^{-\lambda \tau}+g_{2} g_{3}=0\right.
\end{aligned}
$$

where

$$
g_{1}=\varepsilon+\rho+\omega+2 \mu, g_{2}=\varphi+\mu-\beta^{*} \frac{E_{0}^{*}}{N}, g_{3}=(\varepsilon+\mu)(\rho+\omega+\mu) .
$$

If we insert $\lambda=i \omega^{*}$ and $\alpha=1$ in Eq. (4.7), and equating the real and imaginary parts in both sides, then trying to eliminate the variable $\tau$, we have

$$
\left(\omega^{* 2}\right)^{3}+G_{1}\left(\omega^{* 2}\right)^{2}+G_{2} \omega^{* 2}+G_{3}=0,
$$

where

$$
\begin{aligned}
& G_{1}=(\varepsilon+\mu)^{2}+(\rho+\omega+\mu)^{2}+g_{2}^{2}-\sigma^{2}, \\
& G_{2}=g_{3}^{2}-\left(g_{2}+\sigma\right)\left((\varepsilon+\mu)^{2}+(\rho+\omega+\mu)^{2}\right)+\sigma^{2} \frac{E_{0}^{*}}{N}\left(2 \sigma(\rho+\omega+\mu)-2 \rho^{2}-\sigma^{2} \frac{E_{0}^{*}}{N}\right) \\
& G_{3}=g_{3}^{2}\left(g_{2}^{2}-\sigma^{2}\right)+2 g_{3} \sigma^{2} \frac{E_{0}^{*}}{N}\left(\rho^{2}+\sigma(\varepsilon+\mu)\right)-\left(\sigma \frac{E_{0}^{*}}{N}\left(\rho^{2}+\sigma(\varepsilon+\mu)\right)\right)^{2} . .
\end{aligned}
$$

It is clear that when $G_{i}>0, i=1: 3$, there is no real solution for $\omega^{*}$, and the system is asymptotically stable for all time delay. Otherwise, there is a positive solution for $\omega^{*}$ and the critical value of the time delay is given by:

$$
\tau_{2}=\frac{1}{\omega^{*}} \frac{\cos ^{-1}\left(a_{1} a_{3}+a_{2} a_{4}\right)}{a_{1}^{2}+a_{2}^{2}}
$$




$$
\begin{aligned}
& a_{1}=\sigma\left(g_{3}-\frac{E_{0}^{*}}{N}\left(\rho^{2}+\sigma(\varepsilon+\mu)\right)-\omega^{* 2}\right), a_{2}=\sigma \omega^{*}\left(g_{1}-\sigma \frac{E_{0}^{*}}{N}\right), \\
& a_{3}=\omega^{* 2}\left(g_{1}+g_{2}\right)-g_{2} g_{3} \text { and } a_{4}=\omega^{* 3}-\omega^{*}\left(g_{1} g_{2}+g_{3}\right) .
\end{aligned}
$$

and $\omega^{*}$ is a positive square root of the equation $\chi^{3}+G_{1} \chi^{2}+G_{2} \chi+G_{3}=0$.

We can summarize these results in the following theorem.

Theorem 4.2 If the equilibrium point $\dot{P}_{0}^{*}$ of the system (4.1) satisfies the conditions of theorem 3.2, for $\alpha=1, \tau=0$, then by increasing the time delay, it is locally asymptotically stable for $\tau<\tau_{2}$ if $G_{1}$ does not satisfy that $G_{i}>0, \forall i=1: 3$, where $\mathrm{G}_{\mathrm{i}}, i=1: 3$ and $\tau_{2}$ are defined by (4.10-4.12).

Now, following the same procedure, we can state the following theorem.

Theorem 4.3 If the equilibrium point $P_{0}^{*}$ of the system (4.1) satisfy the conditions of theorem (3.2) for $\alpha \in(0,1), \tau=0$, then $P_{0}^{*}$ is locally asymptotically stable for all $\tau>$ 0 if $h_{i}>0, i=1: 5$, and it is locally asymptotically stable for all $\tau<\tau_{3}$, where

$$
\begin{aligned}
& h_{0}=\left(g_{2} g_{3}\right)^{2}-\sigma^{2}\left(g_{3}-\frac{E^{*}}{N}\left(\rho^{2}+\sigma(\varepsilon+\mu)\right)\right)^{2}, \\
& h_{1}=2 \cos \left(\frac{\alpha \pi}{2}\right)\left(\sigma^{2}\left(g_{1}-\frac{\sigma E^{*}}{N}\right)^{2}+g_{2} g_{3}\left(g_{1} g_{2}+g_{3}\right)-\sigma^{2} g_{3}\left(g_{1}-\frac{\sigma E^{*}}{N}\right)\right), \\
& h_{2}=\left(g_{1} g_{2}+g_{3}\right)^{2}-\sigma^{2}\left(g_{1}-\frac{\sigma E^{*}}{N}\right)^{2}-2 \sigma^{2} \cos (\alpha \pi)\left(g_{3}-\frac{E^{*}}{N}\left(\rho^{2}+\sigma(\varepsilon+\mu)\right),\right. \\
& h_{3}=2 \cos \left(\frac{\alpha \pi}{2}\right)\left(\left(g_{1}+g_{2}\right)\left(g_{1} g_{2}+g_{3}\right)-\sigma^{2}\left(g_{1}-\frac{\sigma E^{*}}{N}\right)\right), \\
& h_{4}=2 \cos (\alpha \pi)\left(g_{1} g_{2}+g_{3}\right)+\left(g_{1}+g_{2}\right)^{2}-\sigma^{2}, \\
& h_{5}=2 \cos \left(\frac{\alpha \pi}{2}\right)\left(g_{1}+g_{2}\right) .
\end{aligned}
$$

and is the smallest value of

$$
\tau_{3}=\frac{\ln \left(H_{1}^{2}+H_{2}^{2}\right) /\left(H_{3}^{2}+H_{4}^{2}\right)}{2 r \cos \left(\frac{\alpha \pi}{2}\right)},
$$

where is a positive root of the equation

$$
x^{6}+h_{5} x^{5}+h_{4} x^{4}+h_{3} x^{3}+h_{2} x^{2}+h_{1} x+h_{0}=0
$$

where 


$$
\begin{aligned}
& H_{1}=\sigma\left(r^{2} \cos (\alpha \pi)+r \cos \left(\frac{\alpha \pi}{2}\right)\left(g_{1}-\frac{\sigma E^{*}}{N}\right)+g_{3}-\frac{E^{*}}{N}\left(\rho^{2}+\sigma(\varepsilon+\mu)\right),\right. \\
& H_{2}=\sigma\left(r^{2} \sin (\alpha \pi)+r \sin \left(\frac{\alpha \pi}{2}\right)\left(g_{1}-\frac{\sigma E^{*}}{N}\right),\right. \\
& H_{3}=r^{3} \cos \left(\frac{3 \alpha \pi}{2}\right)+r^{2} \cos (\alpha \pi)\left(g_{1}+g_{2}\right)+r \cos \left(\frac{\alpha \pi}{2}\right)\left(g_{1} g_{2}+g_{3}\right)+g_{2} g_{3}, \\
& H_{4}=r^{3} \sin \left(\frac{3 \alpha \pi}{2}\right)+r^{2} \sin (\alpha \pi)\left(g_{1}+g_{2}\right)+r \sin \left(\frac{\alpha \pi}{2}\right)\left(g_{1} g_{2}+g_{3}\right) .
\end{aligned}
$$

In this part, we consider the epidemic equilibrium point $\dot{p}_{1}$, when the parameters of the system (4.1) satisfy the conditions of theorem 3.3, for $\tau=0$. Now, to evaluate the value of time delay at which the stability of the system will be lost. In this case, we construct the characteristic equation of system (4.1) at the disease equilibrium point $\dot{p}_{1}$.

Let the critical eigenvalue to be $\lambda=r e^{i\left(\frac{\alpha \pi}{2}\right)}$ then, the characteristic equation is

$$
\lambda^{4}+c_{1} \lambda^{3}+c_{2} \lambda^{3} e^{-\lambda \tau}+c_{3} \lambda^{2}+c_{4} \lambda^{2} e^{-\lambda \tau}+c_{5} \lambda e^{-\lambda \tau}+c_{6} \lambda+c_{7} e^{-\lambda \tau}+c_{8}=0,
$$

where

$$
\begin{aligned}
& c_{1}=g_{1}+g_{4}+g_{5}, \quad c_{2}=\sigma, c_{3}=g_{1} g_{4}+g_{3}+\beta\left(g_{5}+\frac{\beta^{*} E_{1}}{N}-\beta_{2} S_{1}\right)+g_{6}\left(g_{1}+g_{4}\right), \\
& c_{4}=\sigma\left(g_{1}+g_{5}-\frac{\sigma E_{1}}{N}\right), c_{5}=\sigma\left(g_{3}-\frac{E_{1}}{N}\left(\rho^{2}+\sigma(\varepsilon+\mu)+g_{6}\left(g_{1}-\frac{\sigma E_{1}}{N}\right)+\beta\left(g_{5}+\frac{\sigma E_{1}}{N}\right)\right),\right. \\
& c_{6}=g_{3} g_{4}+\beta g_{1}\left(g_{5}+\frac{\beta^{*} E_{1}}{N}-\beta_{2} S_{1}\right), \\
& c_{7}=-\sigma\left(g _ { 6 } \left(g_{3}-\frac{E_{1}}{N}\left(\rho^{2}+\sigma(\varepsilon+\mu)\right)+\beta\left((\varepsilon+\mu)\left(g_{5}+\frac{\sigma E_{1}}{N}\right)+\rho\left(g_{5}+\frac{\rho E_{1}}{N}\right)\right),\right.\right. \\
& c_{8}=g_{3}\left(g_{4} g_{6}+\beta\left(g_{5}+\frac{\beta^{*} E_{1}}{N}-\beta_{2} S_{1}\right),\right.
\end{aligned}
$$

with $g_{1}$ and $g_{3}$ given by (4.8) and

$$
g_{4}=\varphi+\mu-\frac{\beta^{*} E_{1}}{N}, g_{5}=\beta_{1} E_{1}+\beta_{2} I_{1}, g_{6}=g_{5}-\beta_{1} S_{1}+\mu+\beta, \beta=\frac{\beta^{*} I_{1}+\sigma R_{1}+\rho U_{1}}{N} .
$$

Theorem 4.4 If the equilibrium point $\stackrel{P}{P}_{1}$ of the system (4.1) satisfy the conditions of theorem (3.3) $\tau=0$, then for $\tau>0, \dot{P}_{1}$ is locally asymptotically stable for, $\tau<\tau_{4}$, if

$$
C_{1}^{2}+C_{2}^{2}>C_{3}^{2}+C_{4}^{2},
$$

where 

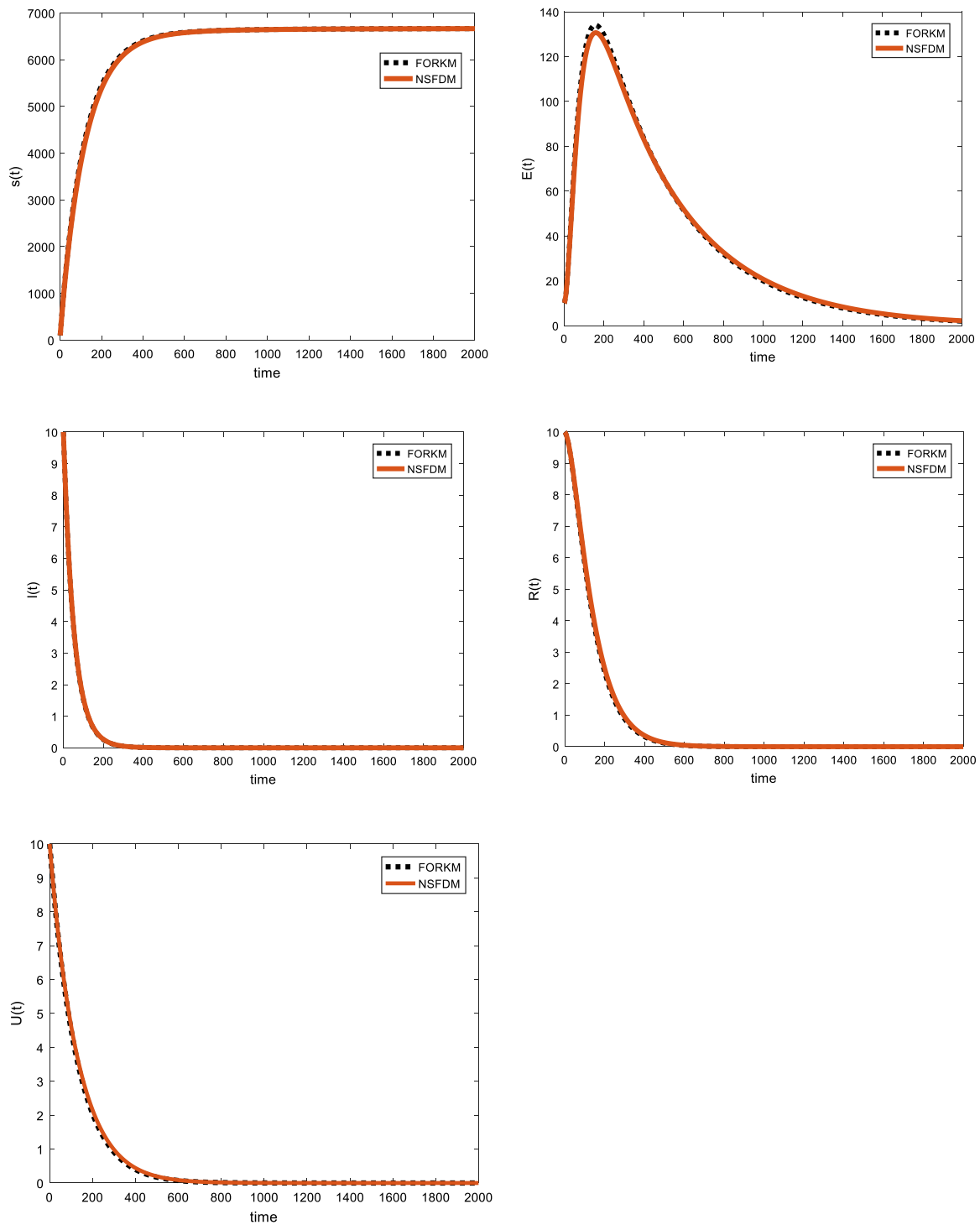

Fig. 2 Time response of $S(t), E(t), I(t), R(t)$ and $U(t)$ in example $1, \alpha=1$

$$
\tau_{4}=\frac{\ln \left(C_{1}^{2}+C_{2}^{2}\right) /\left(C_{3}^{2}+C_{4}^{2}\right)}{2 r \cos \left(\frac{\alpha \pi}{2}\right)},
$$

and 

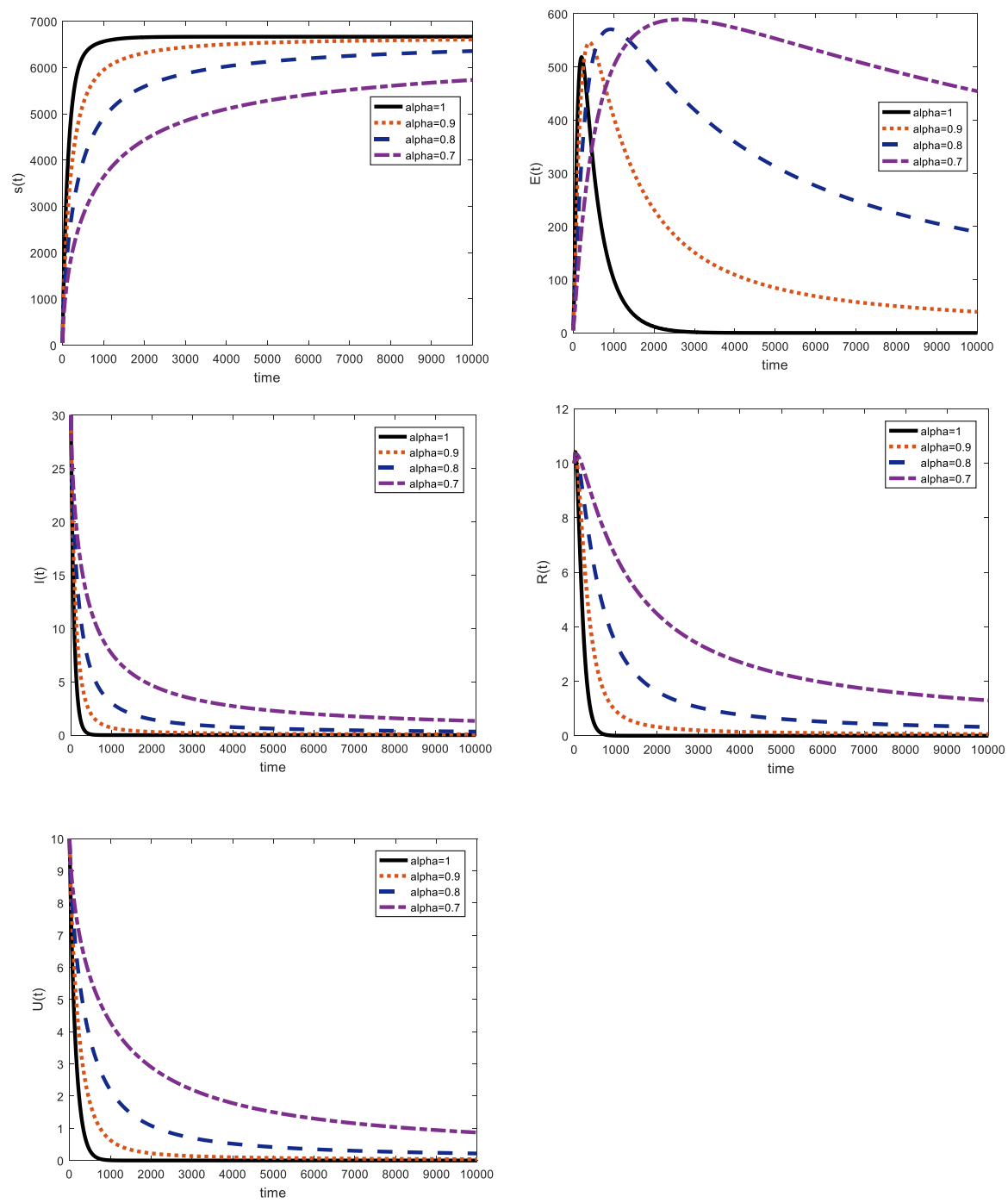

Fig. 3 Time response of $S(t), E(t), I(t), R(t)$ and $U(t)$ in example $1, \alpha=1,0.9,0.8,0.7$

$$
\begin{aligned}
& C_{1}=c_{2} r^{3} \cos \left(\frac{3 \alpha \pi}{2}\right)+c_{4} r^{2} \cos (\alpha \pi)+c_{5} r \cos \left(\frac{\alpha \pi}{2}\right)+c_{7}, \\
& C_{2}=c_{2} r^{3} \sin \left(\frac{3 \alpha \pi}{2}\right)+c_{4} r^{2} \sin (\alpha \pi)+c_{5} r \sin \left(\frac{\alpha \pi}{2}\right), \\
& C_{3}=-\left(r^{4} \cos (2 \alpha \pi)+c_{1} r^{3} \cos \left(\frac{3 \alpha \pi}{2}\right)+c_{3} r^{2} \cos (\alpha \pi)+c_{6} r \cos \left(\frac{\alpha \pi}{2}\right)+c_{8}\right), \\
& C_{4}=-\left(r^{4} \sin (2 \alpha \pi)+c_{1} r^{3} \sin \left(\frac{3 \alpha \pi}{2}\right)+c_{3} r^{2} \sin (\alpha \pi)+c_{6} r \sin \left(\frac{\alpha \pi}{2}\right)\right),
\end{aligned}
$$



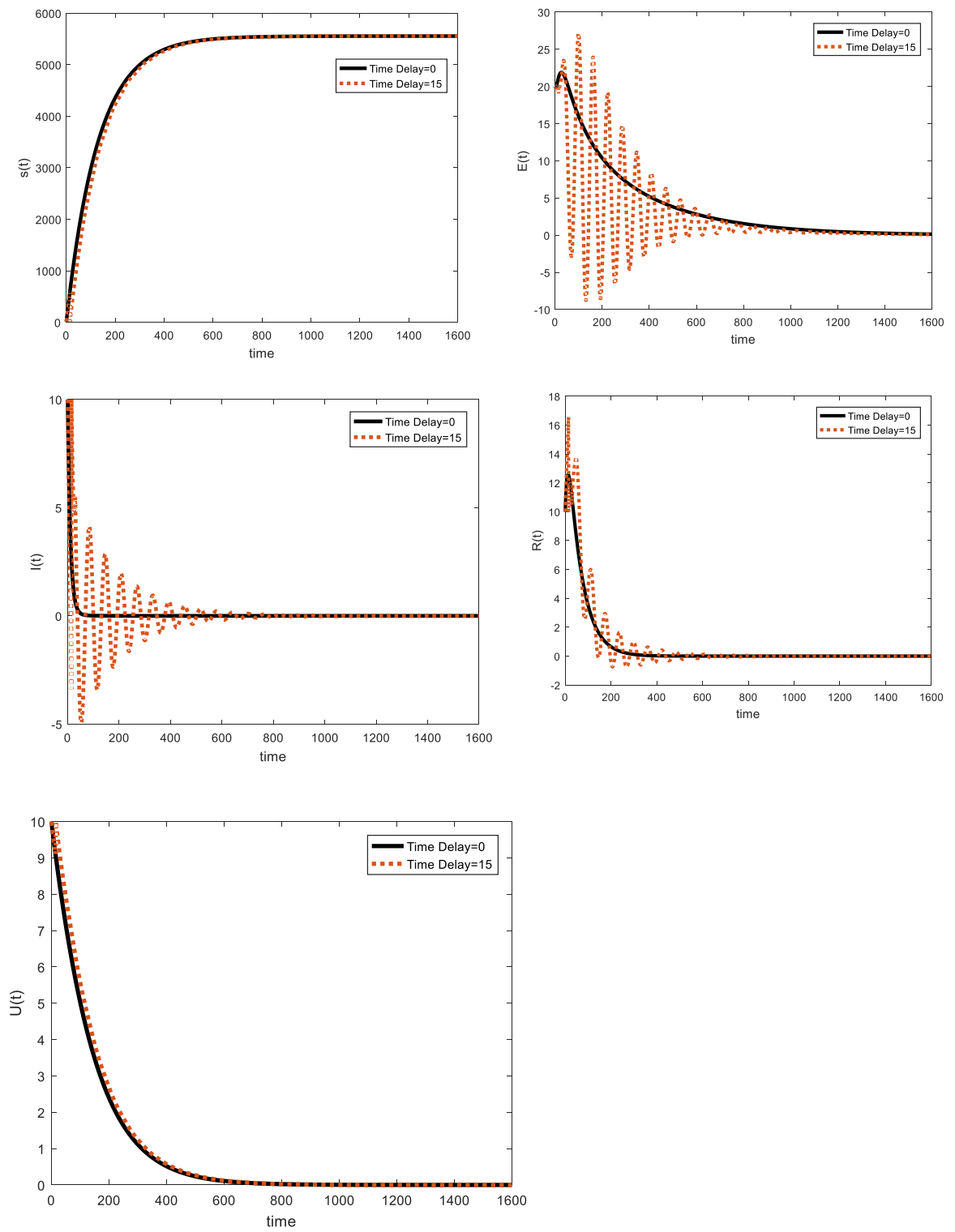

Fig. 4 Time response of $S(t), E(t), I(t), R(t)$ and $U(t)$ in example $2, \tau=0, \tau=15<\tau_{1}$

$c_{j}, i=1: 8$ and are displayed in (4.17), and is the smallest value that satisfies the inequality (4.19).

Proof Equating the real and imaginary parts of Eq. (4.16) by zero, and solving the obtained two equations, we find that $\tau_{4}$ is determined by Eq. (4.20). It is clear that condition (4.19) is the required condition to have a positive real value for $\tau_{4}$. 

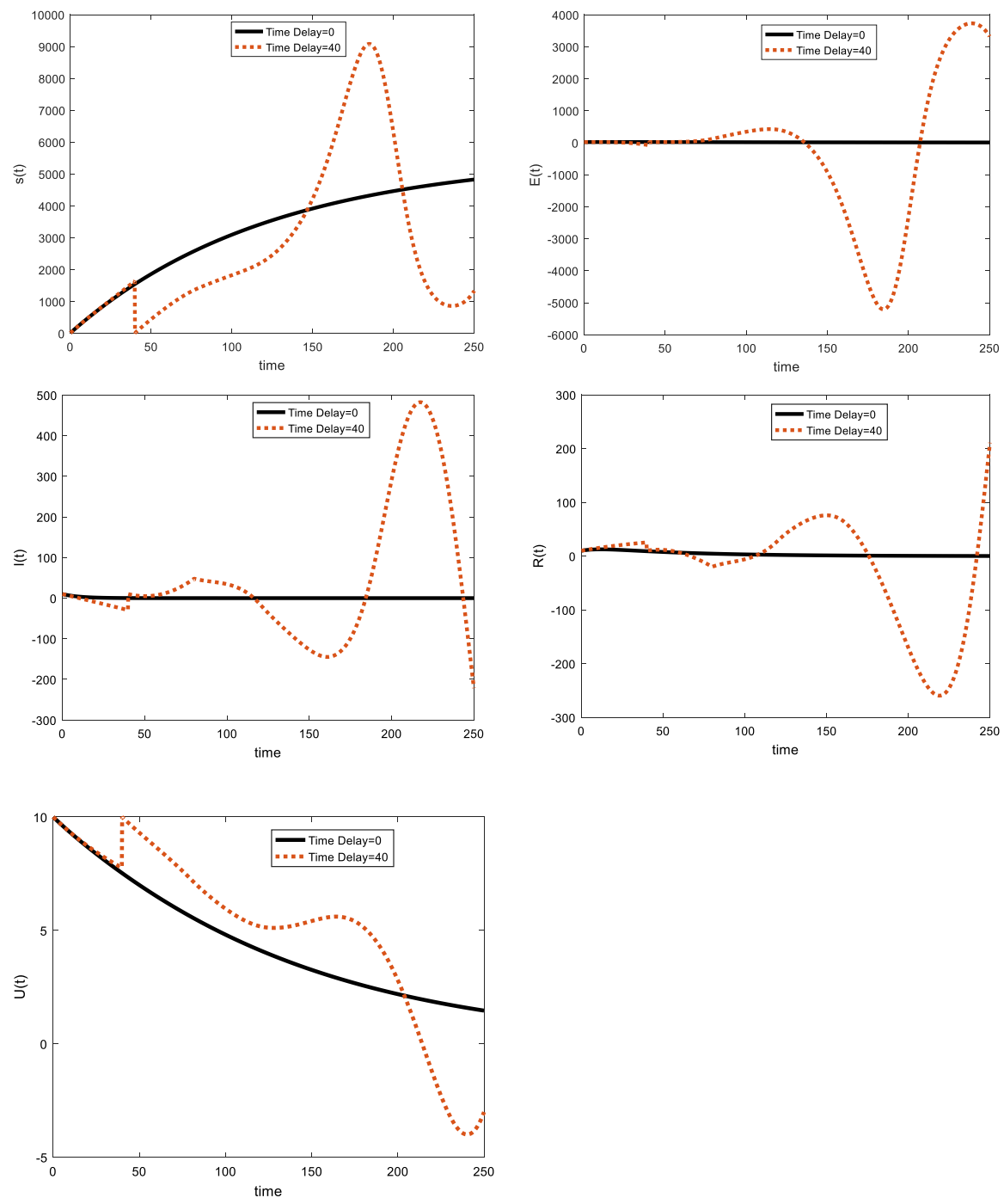

Fig. 5 Time response of in $S(t), E(t), I(t), R(t)$ and $U(t)$ example 2, $\tau=0, \tau=40<\tau_{1}$

\section{Numerical simulation and discussion}

In this section, we give some numerical examples which illustrate the obtained theoretical results and the effect of the system parameters on the stability properties.

Example 1 Assume that the parameters of the system (1.1) have the following values: $\pi=60, \beta_{1}=0.000001, \beta_{2}=0.0002, \beta^{*}=0.0002, \varepsilon=0.000095, \mu=0.009$, $\sigma=0.005, \rho=0.00095, \omega=0.0007$ and $\varphi=0.0005$. Hence $R_{0}=0.74$, the FDEP $p_{0}=(6666,0,0,0,0)$ and it is globally asymptotically stable by Theorem 3.1 for 

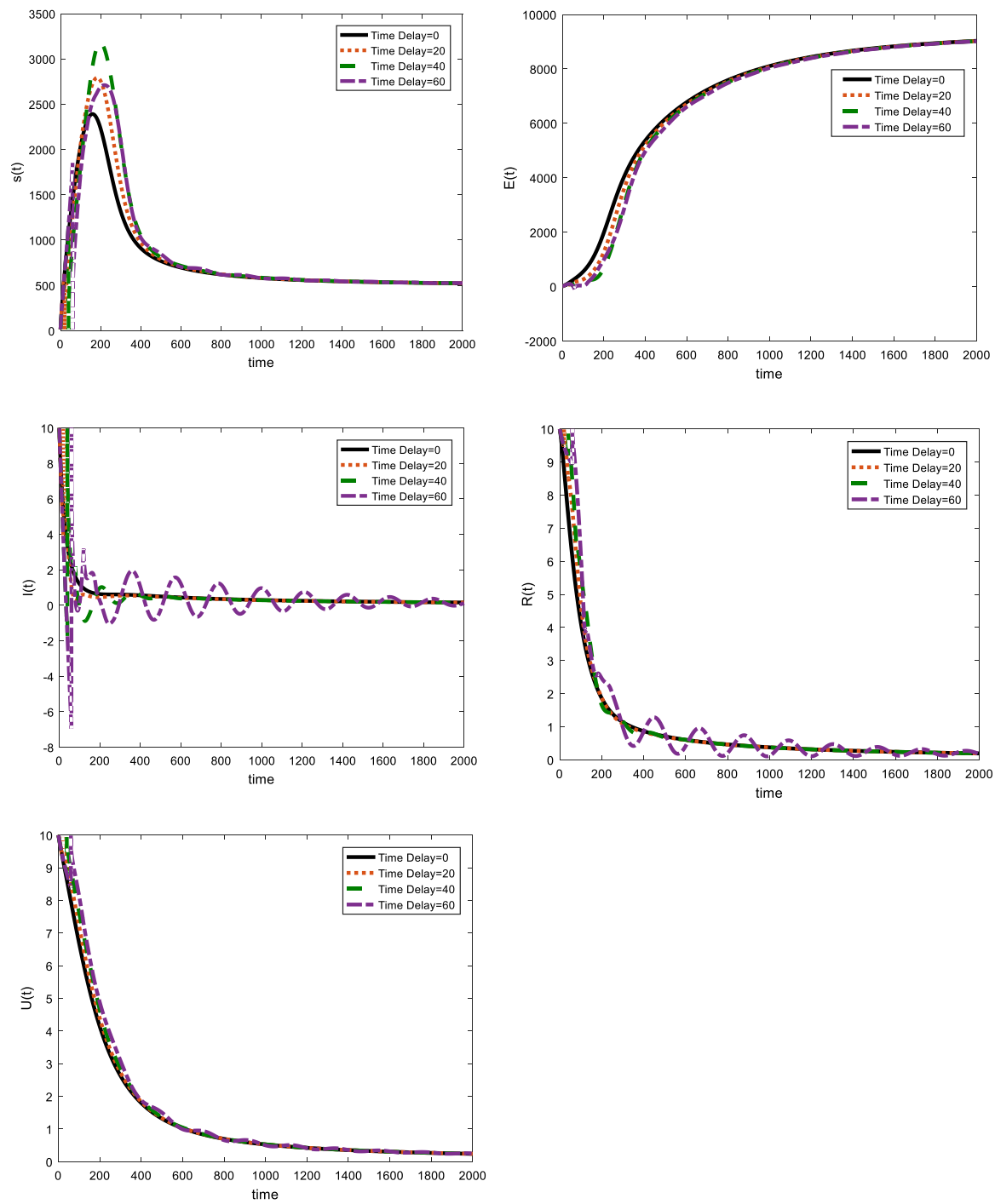

Fig. 6 Time response of $S(t), E(t), I(t), R(t)$ and $U(t)$ in example 3, $\tau=0,20,40$ and 60

$\alpha \in(0,1]$. Figure 2 presents the time response of the system (1.1) for $\alpha=1$, using the fourth-order Rung-Kutta method (FORKM) [26] for solving ordinary differential equations and the nonstandard finite difference method (NSFDM) [23, 27] for solving the fractional-order differential equations. In Fig. 3 the effect of the order of derivative $\alpha$ is shown. We note that the system needs more time interval to reach the equilibrium point $P_{0}$ when $\alpha$ decreases.

Example 2 When the parameters of the system (1.1) have the following values: $\alpha=1, \pi=50, \beta_{1}=0.000001, \quad \beta_{2}=0.0002, \beta^{*}=0.0002, \varepsilon=0.000095, \mu=$ 

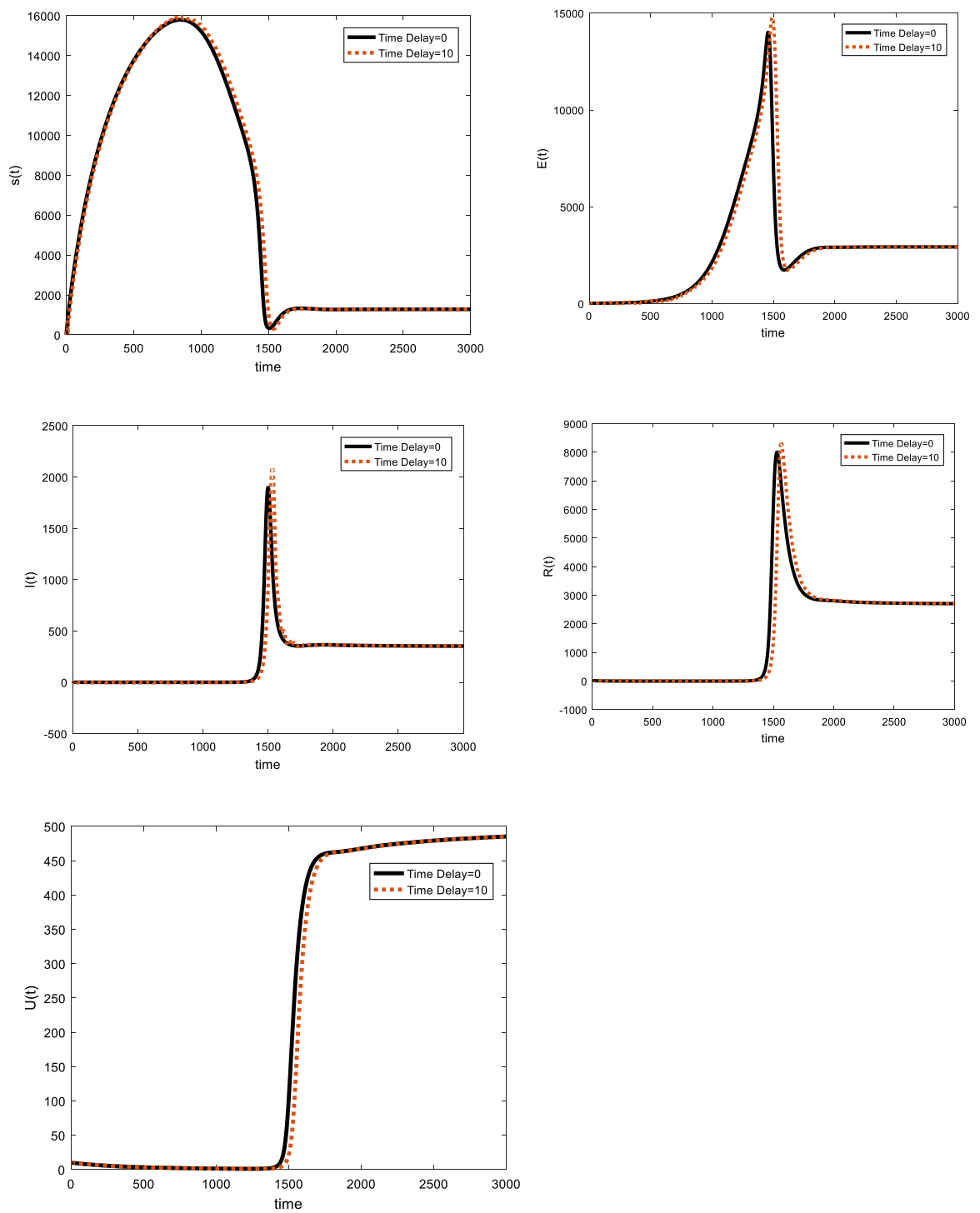

Fig. 7 Time response of $S(t), E(t), I(t), R(t)$ and $U(t)$ in example $4, \tau=0,10$

$0.009, \sigma=0.08, \rho=0.00095, \omega=0.01, \varphi=0.02$. Hence, $R_{0}=0.617$, the FDEP $p_{0}=(5555,0,0,0,0)$ is globally asymptotically stable by Theorem 3.1 when $\tau=0$. By theorem 4.1 the critical value of the time delay, $\tau=37$ and $P_{0}$ becomes unstable if the time delay, $\tau_{1}=37$ is more than $\tau=0$. Figures 4 and 5 illustrate the time response of the system (1.1) for $\tau=15$ and 40 respectively. 

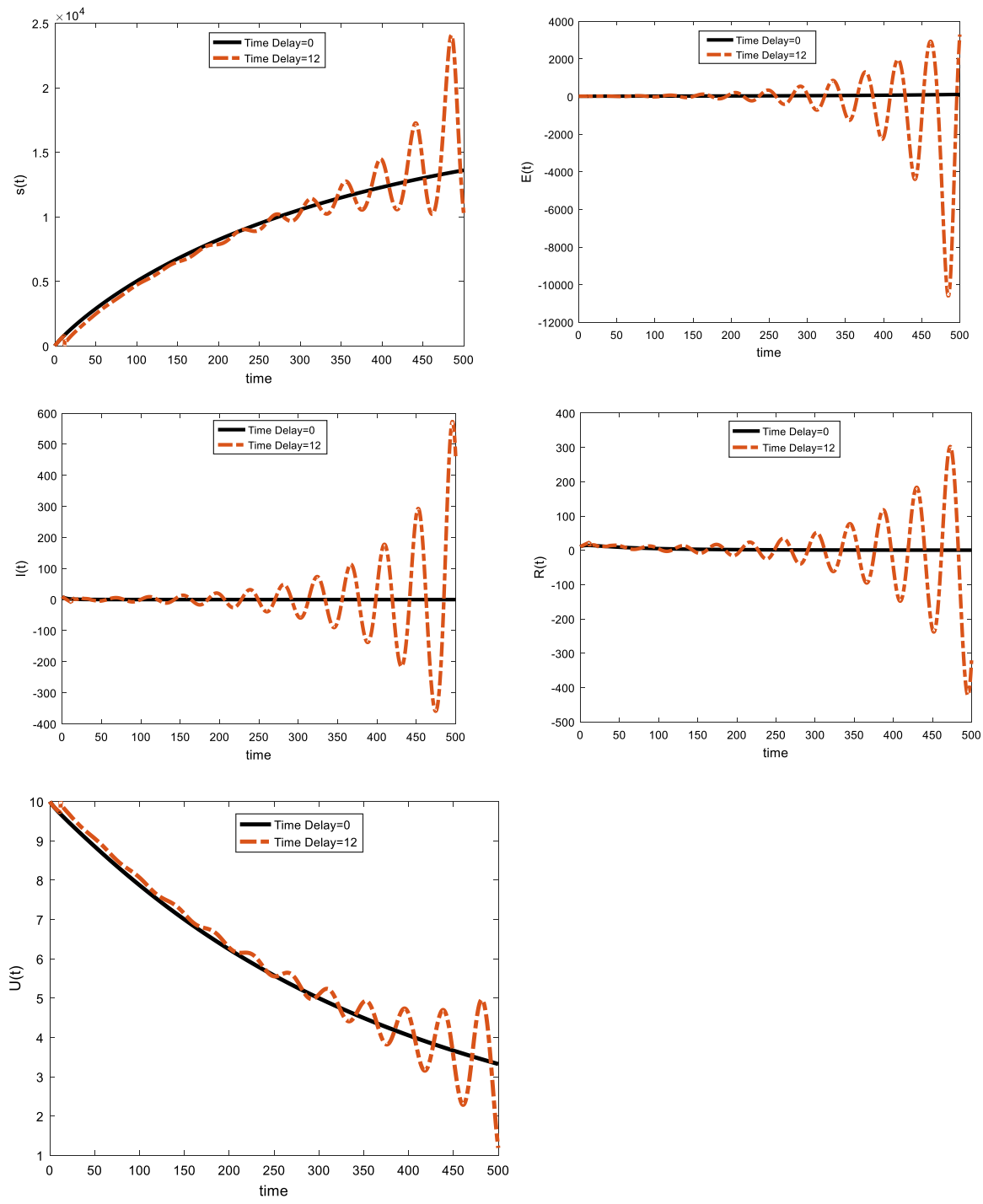

Fig. 8 Time response of $S(t), E(t), I(t), R(t)$ and $U(t)$ in example $4, \tau=0,12$

Example 3 When the parameters of the system (1.1) have the values: $\alpha=0.9, \pi=50$, $\beta_{1}=0.00001, \beta_{2}=0.002, \beta^{*}=0.002, \varepsilon=0.0095, \mu=0.005, \sigma=0.02, \rho=0.0095$, $\omega=0.01, \varphi=0.02$. Hence, $R_{0}=20$, the equilibrium point $p_{0}=(500,9500,0,0,0)$ is asymptotically stable by Theorem 3.2 when. And by Theorem $4.3, P_{0}^{*}$ is asymptotically stable for $\tau>0$. Figure 6 gives the time response of the system (4.1) for $\tau=$ $0,20,40$, and 60 respectively. 
Example 4 When the parameters of system (1.1) are: $\alpha=0.9, \pi=100$, $\beta_{1}=0.000001, \beta_{2}=0.0002, \beta^{*}=0.0002, \varepsilon=0.0000095, \mu=0.005, \sigma=0.02$, $\rho=0.00095, \omega=0.02, \varphi=0.02$. The basic reproduction number $R_{0}=4$, the equilibrium point $P_{1}=(1279,2916,351,2708,505)$ is asymptotically stable by theorem 3.3 when $\tau=0$. However, by Theorem 4.4, $P_{1}$ will be unstable by increasing the time delay. Figures 7 and 8 give the time response of the system (4.1) for $\tau=0,10$ and $\tau=12$ respectively. And it is noted that when the time delay increases the system loses its stability and the disease cannot be controlled. So keeping the infected people quarantined without delay is very important in combating and controlling the spread of the disease.

\section{Conclusion}

There is an urgent need for the efforts of all parties health organizations, governments, and citizens to confront the Corona pandemic, especially since there is no complete cure for this disease. The basic reproduction number $R_{0}$ has been evaluated, where it is shown that the FDEP of the system is globally asymptotically stable when $R_{0}<1$ and unstable when $R_{0}>1$. When taking the necessary precautionary measures that prevent the transmission of infection, especially from uninfected virus carriers (i.e. $\beta_{1}=0$ ) to those in contact with them, especially for individuals with chronic and elderly diseases from the methods of hygiene and the use of masks, the disease can be controlled wonderfully because there is no chance of secondary infection. This fact has been shown by (2.11), where the basic reproduction number $\widetilde{R}=0$. Where there is no incidence rate in the recovered population, the secondary infection by patients with COVID-19 has been clinically declared negative and free from the virus and the virus is completely cleared. The equilibrium points of the system (1.1) are displayed with sufficient conditions that guarantee the asymptotic stability of these points. By numerical simulation, we find that the system needs more interval times to reach the stability position when the order of differentiation $\alpha$ less than the unity as illustrated in example 1 . The rate of quarantine application of the injured, $\sigma$ and the diseaseinduced death rate of the infected population not quarantined $\varphi$ plays an important role in controlling the disease when there is a time delay in applying the injured quarantine strategy as established in theorem 4.1. This is evidenced by the results obtained in the theories (4.1:4.4) where threshold values of the time delay at which the system will lose its stability. So keeping the infected people quarantined immediately is very important in combating and controlling the spread of the disease. It has been evident from the analytical and numerical results obtained in this work that being careful not to delay the implementation of quarantine measures maintains control over the spread of the Corona epidemic. 


\section{References}

1. Yuen, K.S., Ye, Z.W., Fung, S.Y., Chan, C.P., Jin, D.Y.: SARS-CoV-2 and COVID-19: The most important research questions. Cell Biosci. 10, 40 (2020). https://doi.org/10.1186/ s13578-020-00404-4

2. Guo, Y.R., Cao, Q.D., Hong, Z.S., Tan, Y.Y., Chen, S.D., Jin, H.J., Tan, K.S., Wang, D.Y., Yan, Y.: The origin, transmission and clinical therapies on coronavirus disease 2019 (COVID-19) outbreakan update on the status. Milit. Med. Res. 7, 11 (2020). https://doi.org/10.1186/s40779-020-00240-0

3. WHO Coronavirus Disease (COVID-19) Dashboard Data last updated: 2020/10/16, 4:11pm CEST. https://www.who.int/emergencies/diseases/novel-coronavirus-2019

4. WHO Coronavirus Disease (COVID-19), Weakly operational update 14 December 2020, who.int

5. Censolo, R., Morelli, M.: COVID-19 and the potential consequences for social stability. Peace Econ. Peace Sci. Pub. Pol. 26(3), 20200045 (2020)

6. Dias, P.G.I., Rathnayaka, R.M.U.S.K.: Transmission, stability, symptoms, diagnosis and management of COVID 19. Asian J. Res. Infect. Dis. 4(1), 39-47 (2020)

7. Zhang, R., Li, Y., Zhang, A.L., Wang, Y., Molina, M.J.: Identifying airborne transmission as the dominant route for the spread of COVID-19. PNAS 117(26), 14857-14863 (2020)

8. Harapan, H., Itoh, N., Yufika, A., Winardi, W., Keam, S., Te, H., Megawati, D., Hayati, Z., Wagner, A.L., Mudatsir, M.: Coronavirus disease 2019 (COVID-19): a literature review. J. Infect. Public Health 13(2020), 667-673 (2020)

9. Di Gennaro, F., Pizzol, D., Marotta, C., Antunes, M., Racalbuto, V., Veronese, N., Smith, L.: Coronavirus diseases (COVID-19) current status and future perspectives: a narrative review. Int. J. Environ. Res. Public Health 17, 2690 (2020). https://doi.org/10.3390/ijerph17082690

10. Tahir, M., Ali Shah, I.S., Zaman, G., Khan, T.: Prevention strategies for mathematical model MERS-corona virus with stability analysis and optimal control. J. Nanosci. Nanotechnol. Appl. 3(1), 101 (2018)

11. Mohsen, A.A., AL-Husseiny, H.F., Zhou, X., Hattaf, K.: Global stability of COVID-19 model involving the quarantine strategy and media coverage effects. AIMS Public Health 7(3), 587-605 (2020). https://doi.org/10.3934/publichealth.2020047

12. Victor, A.O.: Mathematical predictions for COVID-19 as a global pandemic. Electron. J. (2020). https://doi.org/10.2139/ssrn.3555879

13. World Health Organization COVID 19 Infection. Available online: https://www.who.int/emerg encies/diseases

14. Saeedian, M., Khalighi, M., Azimi-Tafreshi, N., Jafari, G.R., Ausloos, M.: Memory effects on epidemic evolution: the susceptible-infected-recovered epidemic model. Phys. Rev. 95, 022409 (2017)

15. Miller, K.S., Ross, B.: An Introduction to the Fractional Calculus and Fractional Differential Equations. Wiley, New York (1993)

16. Podlubny, I.: Fractional Differential Equations. Technical University of Kosice, Slovak Republic (1999)

17. Mehdi, D., Majid, B.: Application of fractions calculus. Appl. Math. Sci. 4, 1021-1032 (2010)

18. Matignon, D.: stability results for fractional differential equations with applications to control processing. Computat. Eng. Syst. Appl. 2, 963 (1996)

19. Ahmed, E., El-Sayed, A.M.A., El-Saka, H.A.A.: On some Routh-Hurwitz conditions for fractional order differential equations and their Applications in Lorenz, Rossler, Chua and Chen systems. Phys. Lett. 358, 1-4 (2006)

20. El-Sayed, A.M.A., Elsonbaty, A.A., Elsadany, A.E.: Matouk: dynamical analysis and circuit simulation of a new fractional-order hyperchaotic system and its discretization. Int. J. Bifurcat. Chaos 26, 1-35 (2016)

21. Diekmann, O., Heesterbeek, J.A.P., Roberts, M.G.: The construction of next-generation matrices for compartmental epidemic models. J. R. Soc. Interface (2009). https://doi.org/10.1098/rsif.2009.0386

22. Hikal, M.M.: Dynamic properties for A general SEIV epidemic model. Electron. J. Math. Anal. Appl. 2, 26-36 (2014)

23. Hikal, M.M., Zahra, W.K.: On fractional model of an HIV/AIDS with treatment and time delay. Progr. Fract. Differ. Appl. 2, 55-66 (2016)

24. Driessche, V.D., Watmough, P.J.: Reproduction numbers and sub-threshold endemic equilibria for compartmental models of disease transmission. Math. Biosci. 180, 24-48 (2002) 
25. Gelfand, I.M., Kapranov, M.M., Zelevinsky, A.V.: Discriminants, resultant, and multidimensional determinants. Birkhauser, Boston. ISBN 978-0-8176-3660-9 (1994)

26. Butcher, J.C.: Numerical Methods for Ordinary Differential Equations, 2nd edn, pp.137-316 (2008). https://doi.org/10.1002/9780470753767.ch3

27. Zahra, W.K., Hikal, M.M., Taher, A.B.: Stability analysis of an HIV/AIDS epidemic fractional order model with screening and time delay. AASCIT Commun. 2, 41-49 (2015)

Publisher's Note Springer Nature remains neutral with regard to jurisdictional claims in published maps and institutional affiliations. 